\title{
A Search for Optical Line Variability in Narrow-Line Seyfert 1 Galaxies
}

\section{Ester Giannuzzo}

Dipartimento di Astronomia, Università degli Studi di Bologna, Via Zamboni 33, 40126 Bologna, Italy

Giovanna M. Stirpe

Osservatorio Astronomico di Bologna, Via Zamboni 33, 40126 Bologna, Italy

\begin{abstract}
We present the results of a variability study of 'narrow-line Seyfert 1 galaxies': in 10 objects out of 12 the optical line flux changed in one year, with no statistical differences in variability with respect to 'normal' Seyfert 1s. We discuss how models for NLS1s are affected.
\end{abstract}

\section{Introduction}

'Narrow-line Seyfert 1 galaxies' are Seyfert 1 s in which the permitted lines, though broader than the forbidden lines, have widths $\lesssim 1000 \mathrm{~km} \mathrm{~s}^{-1}$ (FWHM). They present Fe II and sometimes high-ionization iron emission and soft X-ray excesses. Some of the possible reasons for such narrow lines are a smaller mass black hole, projection effects related to an anisotropic BLR (e.g., a disk seen pole-on), a larger distance to the center of the observed clouds (e.g. due to obscuration or lack of the innermost clouds, or a globally larger BLR).

No NLS1 has ever been intensively monitored, in spite of the possibility of constraining the models by evaluating the BLR size. We adopted a statistical approach to determine whether variability is common in NLS1s by obtaining two sets of spectra for a sample of NLS1s, separated by one year, checking for broadline variability and comparing the results with those of an existing data-base of normal Seyfert 1s (also monitored on a yearly basis for 15 years).

\section{Observations and results}

The sample comprises 12 objects, observed at the $1.52-\mathrm{m}$ ESO telescope in La Silla (no variability is expected within one observing run); we corrected for differential slit losses through an intercalibration based on forbidden-line fluxes. In 10 objects, we found appreciable flux changes in one or both of the main optical lines ( $\mathrm{H} \alpha$ and $\mathrm{H} \beta$ ) between the two epochs: in particular, 2 NLS1s did not vary, 3 underwent a strong luminosity increase $(\sim 20-40 \%), 4$ displayed marginal variability $(\sim 10-20 \%)$, while 3 objects clearly varied in only one of the lines. Figure 1a shows the two spectra of ESO 012-G21 and the difference 

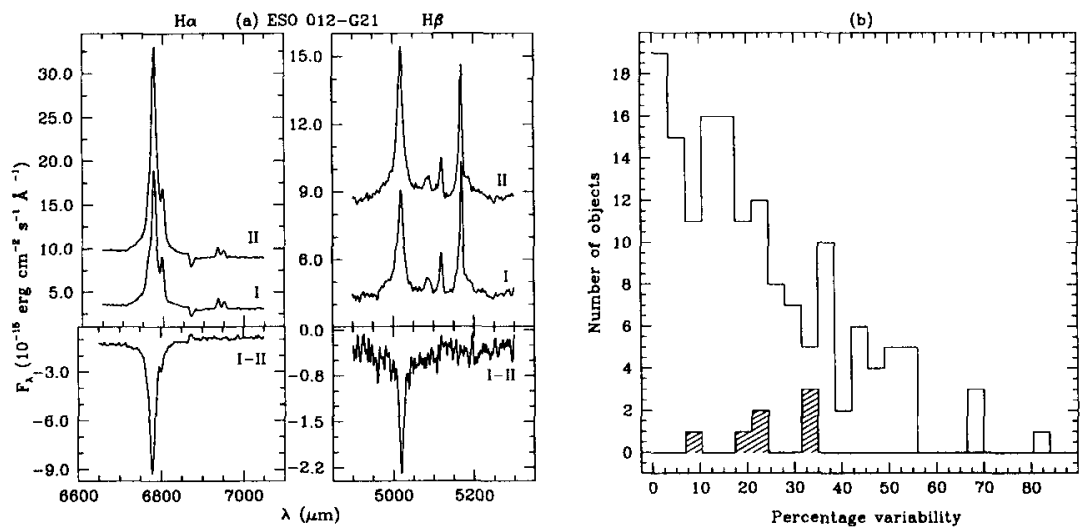

Figure 1. (a) $\mathrm{H} \alpha$ and $\mathrm{H} \beta$ spectra of ESO 012-G21 together with the difference spectra. (b) Comparison between the variability properties of Seyfert 1s (open histogram) and NLS1s (shaded).

spectra for the $\mathrm{H} \alpha$ and the $\mathrm{H} \beta$ regions. The flux variations of the two main Fe II blends are also in broad agreement.

The comparison data set consists of $\sim 20$ normal Seyfert $1 \mathrm{~s}$, monitored at the same telescope. We constructed a histogram including the annual $\mathrm{H} \beta$ relative variations for all objects, representing the global variability behavior of normal Seyfert 1s on a yearly time scale. A similar histogram for NLS1s shows (though with fewer data) no apparent trend of NLS1s towards weak or absent variability. The two histograms are plotted in Figure 1b.

\section{Discussion}

While a lack of variability in NLS1s would have excluded a normal-sized BLR, variability on a one-year time scale does not put a tight constraint on the competing models. However, projection effects due to an anisotropic BLR are improbable, because the small required viewing angle $\left(\lesssim 10^{\circ}\right)$ could not explain the number of existing NLS1s. The obscuration of the inner BLR is also unlikely since the continuum radiation should be obscured too, contrary to the observations; a lack of emitting gas in the innermost BLR should produce very low EWs, while their decrease with FWHM is consistent with a trend shared by the whole Seyfert 1 population. On the other hand, a smaller black hole (mass scaling factor $\lesssim 60$ ) could explain the observed line widths (the possible problem of the different line shape in normal Seyfert 1s and NLS1s may be due to line emissivity distribution effects). Finally, the BLR in NLS1s may be globally larger: our results allow to set an upper limit of $\sim 1$ ly for the region size, since otherwise we would not observe any significant variations.

To discriminate between the two last models, it would be necessary to monitor a variable NLS1 on short time-scales (e.g. once every few days for a few months), since the expected variability properties should be very different (presence or absence of variations) due to the different distance at which the closest emitting clouds should be located (light-days vs. hundreds of light-days). 